\title{
Patients with Epididymo-Orchitis and Meteorological Impact in Taiwan: A Nationwide Population-Based Study
}

\author{
Jui-Ming Liu, ${ }^{1}$ Ying-Hsu Chang, ${ }^{2}$ Te-Wei Ho, ${ }^{3}$ Fung-Wei Chang, ${ }^{4}$ See-Tong Pang, \\ Ren-Jun Hsu, ${ }^{5,6,7}$ and Po-Hung Lin $^{2,8}$ \\ ${ }^{1}$ Division of Urology, Department of Surgery, Taoyuan General Hospital, Ministry of Health and Welfare, Taoyuan, Taiwan \\ ${ }^{2}$ Division of Urology, Department of Surgery, Chang Gung Memorial Hospital, Taoyuan, Taiwan \\ ${ }^{3}$ Graduate Institute of Biomedical Electronics and Bioinformatics, National Taiwan University, Taipei, Taiwan \\ ${ }^{4}$ Department of Obstetrics \& Gynecology, Tri-Service General Hospital, National Defense Medical Center, Taipei, Taiwan \\ ${ }^{5}$ Graduate Institute of Life Sciences, National Defense Medical Center, Taipei, Taiwan \\ ${ }^{6}$ Biobank Management Center, Tri-Service General Hospital, National Defense Medical Center, Taipei, Taiwan \\ ${ }^{7}$ Parasitology, Tri-Service General Hospital, National Defense Medical Center, Taipei, Taiwan \\ ${ }^{8}$ Graduate Institute of Clinical Medical Sciences, College of Medicine, Chang Gung University, Taoyuan, Taiwan
}

Correspondence should be addressed to Ren-Jun Hsu; hsurnai@gmail.com and Po-Hung Lin; honesty1202@gmail.com

Received 6 October 2016; Revised 11 January 2017; Accepted 30 January 2017; Published 20 February 2017

Academic Editor: José Ramón Blanco

Copyright (c) 2017 Jui-Ming Liu et al. This is an open access article distributed under the Creative Commons Attribution License, which permits unrestricted use, distribution, and reproduction in any medium, provided the original work is properly cited.

\begin{abstract}
Background. Epididymo-orchitis is a common infectious disease among men, especially men aged 20 to 39 years. The aim of this study was to analyze possible associations of various meteorological indicators on the incidence of epididymo-orchitis in Taiwan. Methods and Materials. This nationwide population-based study collected data on cases of epididymo-orchitis that were newly diagnosed from 2001 to 2013 in Taiwan. Monthly meteorological indicators, including average temperatures, humidity, rainfall, total rain days, and sunshine hours, were collected from the Central Weather Bureau of Taiwan. Data for a total of 7,233 patients with epididymo-orchitis were collected for this study. Results. The monthly incidence of epididymo-orchitis was positively correlated with temperature, rainfall, and sunshine hours. The average monthly temperature had a linear correlation with the incidence of epididymo-orchitis $(\beta=0.11)$. The monthly average temperature is significantly related, with a positive linear correlation, to the incidence of epididymo-orchitis in Taiwan. Conclusion. This finding may constitute useful information in terms of helping physicians to distinguish between patients with epididymo-orchitis and testicular torsion in hot or cold weather.
\end{abstract}

\section{Introduction}

Epididymo-orchitis consists of inflammation of the epididymis and testes. There are nearly 600,000 cases of epididymoorchitis per year in the United States, accounting for roughly 1/144 (0.69\%) outpatient visits among 18 - to 50-year-old men, with the majority of those patients aged 18 to 35 years [1]. Mittemeyer et al. conducted a study of 610 cases among soldiers in the United States army and their family members and found that most of the patients were aged 20 to 29 years. However, almost all ages were involved (with a range of 4 months to 76 years) [2]. Acute epididymitis is characterized by acute testicular pain and most commonly involves the symptom of acute scrotum as well. Orchitis usually originates from inflammation of the epididymis [3]. The symptoms of epididymo-orchitis include swelling and tenderness of the epididymis or scrotum in about $75 \%$ patients. Some patients even presented with bloodstream infections, sepsis, or septic shock due to the underlying pathogens [4].

Acute scrotum is a symptom that consists of an acute painful swelling of the scrotum. Epididymo-orchitis and testicular torsion should be considered in making a differential diagnosis when the symptom of acute scrotum occurs $[5,6]$. Seasonal variations are known to occur with respect to some infectious diseases and may be due to the epidemiology of the prevalent pathogens, changes in environmental and 
meteorological factors, and alterations in human behavior. For example, seasonal fluctuations in urinary tract infections have been reported in several studies $[7,8]$. The present study sought to evaluate the effect of various meteorological indicators on the incidence of epididymo-orchitis. More specifically, we conducted a 14 -year population-based study to evaluate the relationship between meteorological indicators and epididymo-orchitis in Taiwan.

\section{Methods}

2.1. Data Source. This study is a nationwide populationbased investigation that utilized data from Taiwan's National Health Insurance Research Database (NHIRD). The data in the NHIRD comes from the National Health Insurance (NHI) program, which began in 1995 and covered $99.9 \%$ of Taiwan's 23 million residents as of the end of 2013 [9]. All the medical claims data of inpatients and outpatients are included in the NHIRD. More specifically, this study utilized the Longitudinal Health Insurance Database 2000 (LHID2000) [10], a subdataset of the NHIRD. The LHID2000 includes data from January 2000 to December 2013 for a randomly selected sample of one million people out of the 23 million people included in the NHIRD in the year 2000. The sample of patients included in the LHID2000 has a similar demographic distribution and origin to the broader population included in the NHIRD [11]. All the clinical diagnoses in this study were made according to the International Classification of Diseases, 9th revision, Clinical Modification (ICD-9-CM).

The meteorological data utilized in this study was provided by Taiwan's Central Weather Bureau (CWB) and consists of data collected from $27 \mathrm{CWB}$ weather stations distributed across various territories of Taiwan (i.e., the islands of Taiwan, Penghu, Kinmen, and Lienchiang). The monthly meteorological data included temperature (measured in degree Celsius), relative humidity (measured in percentage), total rainfall amount (measured in millimeters), total rain days (measured in days), and total sunshine hours (measured in hours) [12]. According to the weather typical of Taiwan, the months of March, April, and May constitute the spring season; June, July, and August constitute the summer; September, October, and November are the fall; and December, January, and February comprise winter.

2.2. Ethics Statement. This study was conducted after we received approval from the Institutional Review Board of Chang Gung Memorial Hospital at Linkou (CGMH IRB 103-2071B). As this was a retrospective study and all data was anonymous, the Institutional Review Board department agreed with the authors that it was not necessary to obtain patient consent.

2.3. Study Subjects. The study subjects consisted of patients included in the LHID2000 who were newly diagnosed with epididymitis and orchitis (ICD-9-CM: 604), received a prescription for antibiotic medication, and received testicular sonography examination between January 2000 and December 2013 from the LHID2000 (Figure 1). More specifically, data for patients from administrative regions without CWB weather stations were excluded. In addition, patients who were diagnosed before December 31st, 2000, or after January 1 st, $2013(n=438)$, and patients with incomplete demographic data $(n=1,645)$ were also excluded. Finally, data for a total of 7,223 patients with epididymo-orchitis were collected and analyzed in this study. The diagnoses of epididymoorchitis were based on detailed clinical examinations, with typical symptoms including painful swelling of the scrotum that may radiate to the lower abdomen, fever, frequency and urgency in voiding, and dysuria. The diagnoses of epididymoorchitis were made by urologists, infectious disease physicians, or licensed physicians. All the patients included in this study were under antibiotic treatment.

2.4. Statistical Analysis. Descriptive statistics for the characteristics of study subjects and meteorological data were first calculated by Student's $t$-test and Chi-square test; Spearman's rank correlation was used to examine the relationship between the meteorological factors and the monthly incidence rates of epididymo-orchitis. The linear regression model was also used to estimate the relationship between the meteorological factors and monthly incidence rates of epididymo-orchitis. All the tests were two-sided, with $p$ value $<0.05$ being regarded as statistically significant. All the statistical analyses were performed with SAS 9.2 software.

\section{Results}

Data for a total of 7,223 patients with epididymo-orchitis was collected and analyzed in this 14-year nationwide population-based study. The demographic characteristics of those patients are listed in Table 1. The mean age of male patients with epididymo-orchitis was $43.46 \pm 20.03$ years, with a major proportion being aged 20-49 years. Most of the patients lived in Northern and suburban areas of Taiwan.

The average monthly incidence rate of epididymoorchitis was 9.09 per 100,000 population. A comparison of the average monthly epididymo-orchitis incidence rate with the monthly average meteorological factors is shown in Table 2. During the study period from 2000 to 2013, the hottest month was July, with an average temperature of 28.72 degrees Celsius, while the coolest month was January, with an average temperature of 16.90 degrees Celsius. In addition, the month with the most sunshine hours was July, with an average of 223.84 hours, while the month with the least sunshine hours was February, with an average of 125.81 hours. The relative humidity was highest in June, with an average of $79.31 \%$, and the lowest in December, with an average of $73.73 \%$. The highest average total rainfall was in August, with $347.25 \mathrm{~mm}$, while the lowest average total rainfall was in January, with $62.23 \mathrm{~mm}$. The highest average number of rain days was in June, with 13.80 days, and the lowest average number of rain days was in December, with 7.39 days. The monthly incidence of epididymo-orchitis was highest in May and lowest in February (Table 2). The monthly epididymo-orchitis incidence rate and corresponding monthly meteorological factors during the study period are shown in Figure 1.

The correlations between the meteorological factors and the incidence rate of epididymo-orchitis are listed in Table 3. 


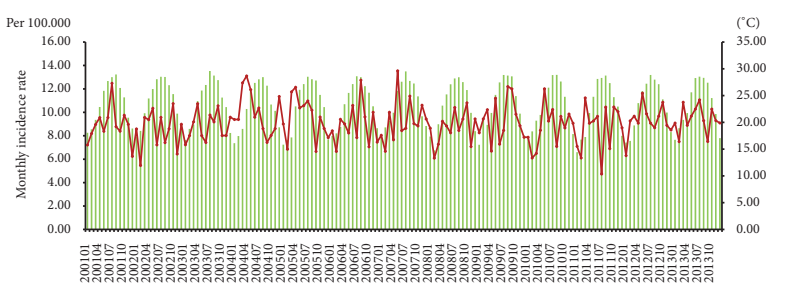

Time

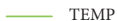

$\longrightarrow$ Monthly incidence rate

(a)

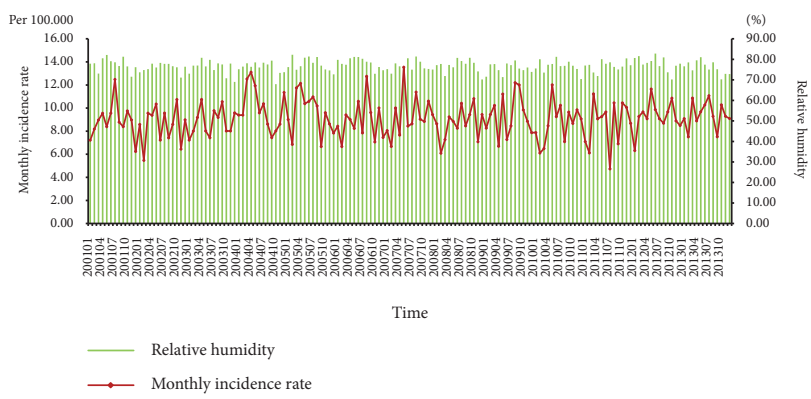

(c)

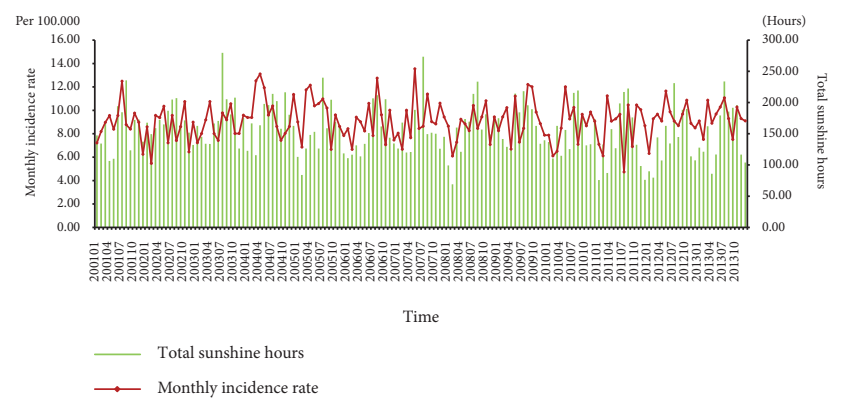

(b)

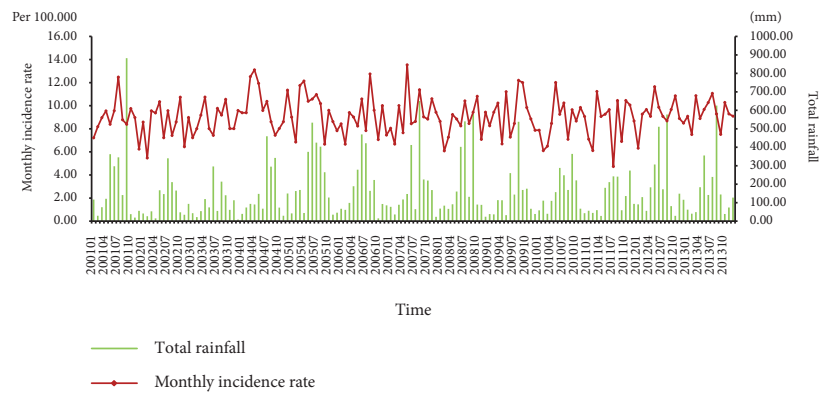

(d)

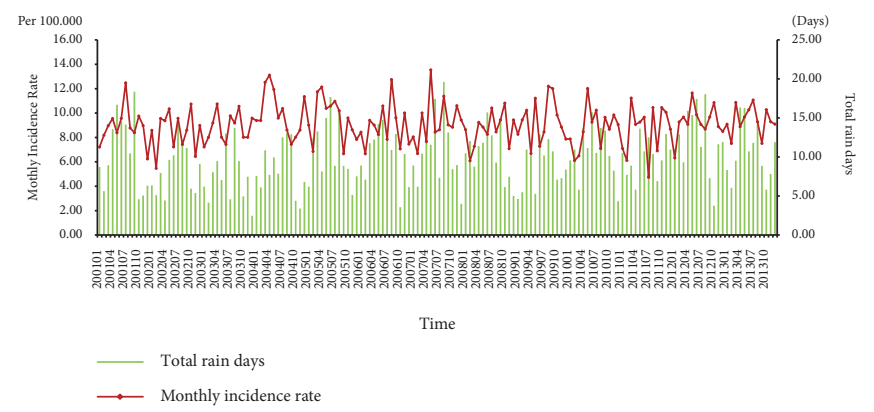

(e)

FIGURE 1: The monthly incidence of epididymo-orchitis and the corresponding monthly weather data during the study period. (a) Epididymoorchitis incidence and monthly average temperature. (b) Epididymo-orchitis incidence and monthly total sunshine hours. (c) Epididymoorchitis incidence and monthly relative humidity. (d) Epididymo-orchitis incidence and monthly total rain fall. (e) Epididymo-orchitis incidence and monthly total rain days.

The average temperature, total rainfall amount, and total sunshine hours had statistically significant correlations with the incidence of epididymo-orchitis. However, only average temperature $(B=0.11, P<0.001)$ was found to have a significant linear correlation with the incidence of epididymoorchitis after regression model analysis (Table 4).

\section{Discussion}

This is the first study to investigate the relationships between different meteorological indicators and the incidence of epididymo-orchitis in Taiwan. The results of this large nationwide population-based study demonstrate that the incidence of epididymo-orchitis is positively correlated with average temperature.

In this study, average temperature $(B=0.11, P<$ 0.001 ) was found to have a significant linear correlation with the incidence of epididymo-orchitis. Lyronis et al. conducted a study of acute scrotum and found that epididymoorchitis was the most common cause of acute scrotum and that the incidence of epididymo-orchitis was higher in summer [13]. Our study also revealed that the incidence rates of epididymo-orchitis were higher in May and July. The retrograde ascent of pathogens is the usual cause of epididymo-orchitis. Sexually transmitted Neisseria gonorrhoeae or Chlamydia trachomatis pathogens are the most common causes of epididymo-orchitis in men aged 14 to 35 years, whereas Escherichia coli pathogens along with urinary tract infection constitute the most common cause in men younger than 14 years and older than 35 years of age [14-17].

Potential mechanisms for the positive correlation between temperature and epididymo-orchitis infection are discussed below. Increased temperatures increase people's perspiration and total body water loss [18]. In turn, the 
TABle 1: Demographic characteristics of study subjects of epididymo-orchitis from 2000 to 2013 in Taiwan.

\begin{tabular}{lc}
\hline Characteristics & $n(\%)$ \\
\hline Number of cases & 7,223 \\
Mean age (years) (standard deviation) & $43.46(20.03)$ \\
Age at diagnosis (years) & \\
$<20$ & $751(10.40)$ \\
$20-29$ & $1,281(17.74)$ \\
$30-39$ & $1,371(18.98)$ \\
$40-49$ & $1,240(17.17)$ \\
$50-59$ & $922(12.76)$ \\
$60-69$ & $657(9.10)$ \\
$\geq 70$ & $1,001(13.85)$ \\
Insured region & \\
Northern & $3,811(52.76)$ \\
Central & $1,153(15.96)$ \\
Southern & $2,056(28.46)$ \\
Eastern & $203(2.82)$ \\
Urbanicity & \\
Urban & $2,623(36.31)$ \\
Suburban & $4,105(56.84)$ \\
Rural & $484(6.70)$ \\
Missing & $11(0.15)$ \\
\hline
\end{tabular}

resulting phenomena of relative dehydration and more concentrated urine with less frequent voiding increase the rate of urinary tract infections. Increased temperatures also cause peripheral vasodilation and the pooling of blood in the skin, leading to a decrease in the effective blood volume. A hot environment may also cause peripheral vasodilation and pooling of blood in the skin which means that exposure to heat can cause a decrease in effective blood volume. In contrast, exposure to cold has also been found to cause changes in the vasopressin system leading to diuresis and the increased clearance of potential pathogens of the urinary tract [19]. In addition, seasonal variations in gonorrhea and Chlamydia infections in adolescents have previously been reported, including higher positive tests rates in the summer and fall [20].

Epididymo-orchitis and testicular torsion are both accompanied by acute scrotal pain or swelling of the scrotum. Differential diagnosis between these two diseases is important due to possibility of torsion resulting in testicular infarction, which constitutes a surgical emergency. The seasonality of testicular torsion has previously been documented in several studies. Chiu et al. conducted a 10 -year cohort study with 1,782 testicular torsion patients that found that January had a significantly higher incidence rate of torsion. In addition, the incidence rate of testicular torsion was negatively associated with temperature [21]. Relatedly, Mabogunje reported an increased incidence of testicular torsion between November and February [22]. Several studies have also reported increased incidences of testicular torsion during low temperature periods in Japan, the United
States, Greece, and Nigeria [13, 22-24]. In contrast, the current study found that the incidence of epididymo-orchitis was increased in May and July, while a positive correlation between epididymo-orchitis and temperature was also noted. These opposing seasonality characteristics of epididymoorchitis and testicular torsion may be helpful in distinguishing the two diseases from each other.

A study conducted in the United State found that nearly 60,000 male adults made visits to office-based physicians for epididymo-orchitis, accounting for $1 / 350(0.29 \%)$ of all the visits in the period under consideration [25]. In another nationwide study in the United States, epididymo-orchitis accounted for 1/144 (0.69\%) of outpatient visits among 18to 50 -year-old men [1]. The 9.09 per 100,000 population incidence of epididymo-orchitis found in the current study was lower than previous studies. The population resident in the area without CWB weather stations was excluded for having no meteorological data available. Some epididymoorchitis patients among this population may be excluded also. This may affect the calculated incidence of epididymoorchitis.

The general climate is Marine Subtropical or tropical climate in Taiwan with a relative small change of temperature between summer and winter. Ratkowsky et al. demonstrated a linear relationship between the growth rate of bacterial culture and temperature even within a small range of temperature in the laboratory [26]. Thus, temperature, even a relative small range of temperature, plays an important role in growth of bacteria.

The strength of the current study is that it utilized a longitudinal nationwide database with large number of subjects and a long follow-up period. However, the study also had several limitations that should also be discussed. First, the diagnosis of epididymo-orchitis was recognized according to the ICD-9 code. The ICD-9-CM code 604.90 is used for both epididymitis and orchitis. Thus, it is difficult to distinguish epididymitis from orchitis using the ICD-9 code. Second, data from diagnostic laboratory tests that can help confirm diagnoses of epididymitis and orchitis, such as urinalysis, urine culture, and C-reactive protein (CRP) level data, are not included in the NHIRD. Therefore, epididymoorchitis caused by sexually transmitted diseases pathogens may be included in this study. The detailed reports of color Doppler ultrasonography are also not included. Third, the data regarding the meteorological indicators came from 27 weather stations monitored by Taiwan's CWB, but these 27 stations did not cover every territory of Taiwan. Finally, this study utilized a retrospective study design. Further prospective studies are thus warranted to investigate the relationship between weather and epididymo-orchitis.

\section{Conclusion}

In conclusion, various meteorological indicators, especially average temperature, appeared to significantly affect the incidence of epididymo-orchitis in Taiwan. A significant positive linear correlation was discovered between temperature and patients with epididymo-orchitis. This result may serve as 


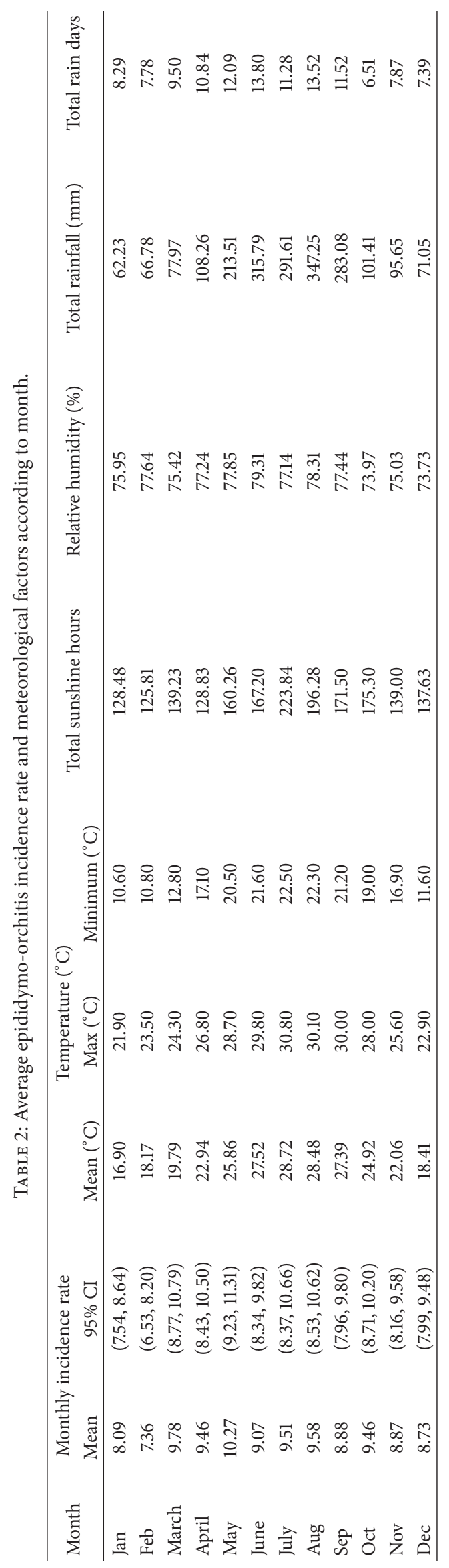


TABLE 3: Spearman's rank correlations between meteorological factors and monthly epididymo-orchitis incidence rates.

\begin{tabular}{lc}
\hline Variable & $r$ \\
\hline Temperature & $0.28^{* * *}$ \\
Total sunshine hours & $0.17^{*}$ \\
Relative humidity & 0.08 \\
Total rainfall & $0.23^{* *}$ \\
Total rain days & 0.14 \\
\hline
\end{tabular}

${ }^{* * *} P<0.001,{ }^{* *} P<0.01,{ }^{*} P<0.05$.

TABLE 4: Regression model of meteorological factors and monthly epididymo-orchitis incidence rates.

\begin{tabular}{lc}
\hline Variable & $\beta$ \\
\hline Temperature & $0.11^{* * *}$ \\
Total sunshine hours & $0.01^{*}$ \\
Relative humidity & 0.04 \\
Total rainfall & $0.002^{*}$ \\
Total rain days & 0.06 \\
\hline
\end{tabular}

ß: coefficient of regression analysis.

${ }^{* * *} P<0.001,{ }^{*} P<0.05$.

a useful clinical clue to help physicians distinguish between patients with epididymo-orchitis and testicular torsion in hot or cold weather.

\section{Competing Interests}

The authors declare that they have no conflict of interests.

\section{Authors' Contributions}

Jui-Ming Liu wrote the manuscript and conducted the data analysis. Fung-Wei Chang and See-Tong Pang wrote the proposal and designed the manuscript. Ying-Hsu Chang and Te-Wei Ho contributed to the conception of the study. Ren-Jun Hsu made data analysis. Po-Hung Lin revised the manuscript. All authors contributed to data analysis, drafting, and critically revising the paper, read and approved the final manuscript, and agreed to be accountable for all aspects of the work. Ren-Jun Hsu and Po-Hung Lin contributed equally to this work.

\section{Acknowledgments}

The authors acknowledge support from the Taiwan Typhoon and Flood Research Institute, National Applied Research Laboratories, which provided data from the Data Bank for Atmospheric \& Hydrologic Research. This work was also supported by grants from the Taoyuan General Hospital, Ministry of Health and Welfare (10322), and Ministry of Science and Technology, Taiwan (MOST 104-2320-B-016012-MY3).

\section{References}

[1] National Center for Health Statistics, "National Ambulatory Medical Care Survey, 2002," http://www.cdc.gov/nchs/about/ major/ahcd/ahcd1.htm.

[2] B. T. Mittemeyer, K. W. Lennox, and A. A. Borski, "Epididymitis: a review of 610 cases," Journal of Urology, vol. 95, no. 3, pp. 390$392,1966$.

[3] G. Luzzi and T. O’Brien, “Acute epididymitis," BJU International, vol. 87, no. 8, pp. 747-755, 2001.

[4] M. Ludwig, "Diagnosis and therapy of acute prostatitis, epididymitis and orchitis," Andrologia, vol. 40, no. 2, pp. 76-80, 2008.

[5] M. Davenport, "ABC of general surgery in children: acute problems of the scrotum," BMJ, vol. 312, no. 7028, pp. 435-437, 1996.

[6] R. Rabinowitz and W. C. Hulbert Jr., "Acute scrotal swelling," Urologic Clinics of North America, vol. 22, no. 1, pp. 101-105, 1995.

[7] J. E. Anderson, "Seasonality of symptomatic bacterial urinary infections in women," Journal of Epidemiology and Community Health, vol. 37, no. 4, pp. 286-290, 1983.

[8] M. E. Falagas, G. Peppas, D. K. Matthaiou, D. E. Karageorgopoulos, N. Karalis, and G. Theocharis, "Effect of meteorological variables on the incidence of lower urinary tract infections," European Journal of Clinical Microbiology and Infectious Diseases, vol. 28, no. 6, pp. 709-712, 2009.

[9] T.-M. Cheng, "Reflections on the 20th anniversary of Taiwan's single-payer national health insurance system," Health Affairs, vol. 34, no. 3, pp. 502-510, 2015.

[10] F.-W. Chang, W.-Y. Lee, Y.-P. Liu et al., "The relationship between economic conditions and postpartum depression in Taiwan: a nationwide population-based study," Journal of Affective Disorders, vol. 204, pp. 174-179, 2016.

[11] National Health Research Institutes, "National Health Insurance Research Database," http://nhird.nhri.org.tw/en/Data Subsets.html\#.

[12] J. Liu, H. Wang, F. Chang et al., "The effects of climate factors on scabies. A 14-year population-based study in Taiwan," Parasite, vol. 23, article 54, 2016.

[13] I. D. Lyronis, N. Ploumis, I. Vlahakis, and G. Charissis, "Acute scrotum -etiology, clinical presentation and seasonal variation," Indian Journal of Pediatrics, vol. 76, no. 4, pp. 407-410, 2009.

[14] K. Manavi, K. Turner, G. R. Scott, and L. H. Stewart, "Audit on the management of epididymo-orchitis by the Department of Urology in Edinburgh," International Journal of STD and AIDS, vol. 16, no. 5, pp. 386-387, 2005.

[15] T. R. Redfern, P. J. English, C. D. Baumber, and D. McGhie, "The aetiology and management of acute epididymitis," British Journal of Surgery, vol. 71, no. 9, pp. 703-705, 1984.

[16] R. Berger, E. Alexander, C. Harnisch Paulsen, G. Monda, J. Ansell, and K. Holmes, "Etiology, manifestations and therapy of acute epididymitis: prospective study of 50 cases," The Journal of Urology, vol. 121, pp. 750-754, 1979.

[17] W. Hoppner, T. Strohmeyer, M. Hartmann, D. Lopez-Gamarra, and K. Dreikorn, "Surgical treatment of acute epididymitis and its underlying diseases," European Urology, vol. 22, no. 3, pp. 218-221, 1992.

[18] B. Melin, N. Koulmann, C. Jimenez et al., "Comparison of passive heat or exercise-induced dehydration on renal water and electrolyte excretion: the hormonal involvement," European Journal of Applied Physiology, vol. 85, no. 3-4, pp. 250-258, 2001. 
[19] Z. Sun, "Genetic AVP deficiency abolishes cold-induced diuresis but does not attenuate cold-induced hypertension," American Journal of Physiology-Renal Physiology, vol. 290, no. 6, pp. F1472-F1477, 2006.

[20] B. Schroeder, P. Tetlow, J. S. Sanfilippo, and S. P. Hertweck, "Is there a seasonal variation in gonorrhea and chlamydia in adolescents?" Journal of Pediatric and Adolescent Gynecology, vol. 14, no. 1, pp. 25-27, 2001.

[21] B. Chiu, C.-S. Chen, J. J. Keller, C.-C. Lin, and H.-C. Lin, "Seasonality of testicular torsion: a 10-year nationwide population based study," Journal of Urology, vol. 187, no. 5, pp. 1781-1785, 2012.

[22] O. A. Mabogunje, "Testicular torsion and low relative humidity in a tropical country," British Medical Journal, vol. 292, no. 6517, pp. 363-364, 1986.

[23] H. Hoshino, T. Abe, H. Watanabe, Y. Katsuoka, and N. Kawamura, "Correlation between atmospheric temperature and testicular torsion," Acta Urologica Japonica, vol. 39, no. 11, pp. 1031-1033, 1993.

[24] A. K. Srinivasan, J. Freyle, J. S. Gitlin, and L. S. Palmer, "Climatic conditions and the risk of testicular torsion in adolescent males," Journal of Urology, vol. 178, no. 6, pp. 2585-2588, 2007.

[25] M. M. Collins, R. S. Stafford, M. P. O’Leary, and M. J. Barry, "How common is prostatitis? A national survey of physician visits," Journal of Urology, vol. 159, no. 4, pp. 1224-1228, 1998.

[26] D. Ratkowsky, J. Olley, T. McMeekin, and A. Ball, "Relationship between temperature and growth rate of bacterial cultures," Journal of Bacteriology, vol. 149, no. 1, pp. 1-5, 1982. 


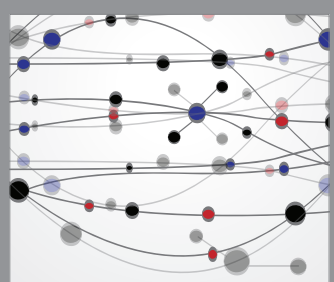

The Scientific World Journal
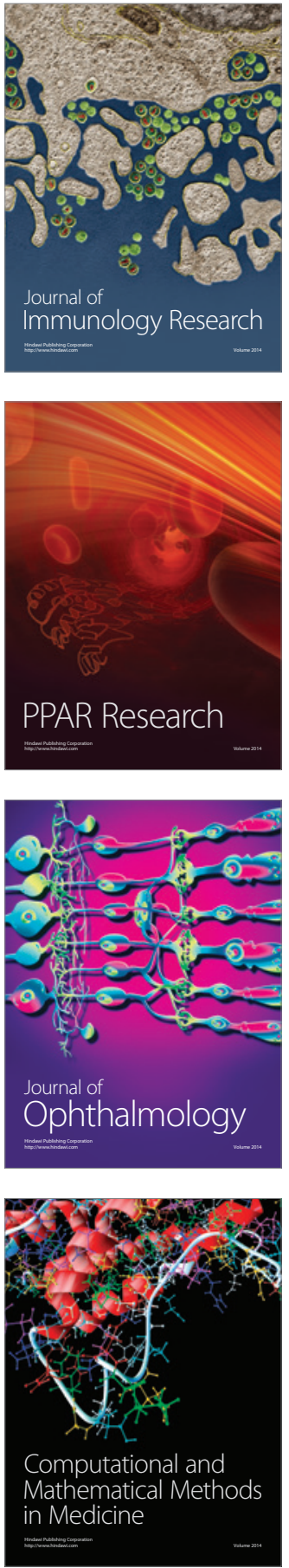

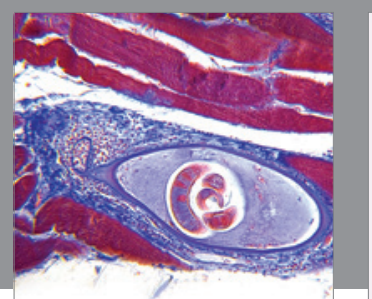

Gastroenterology Research and Practice
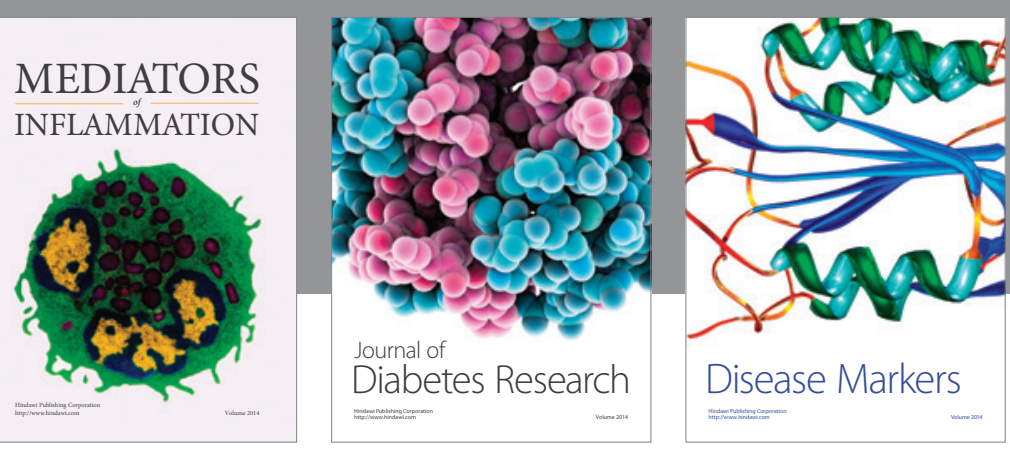

Disease Markers

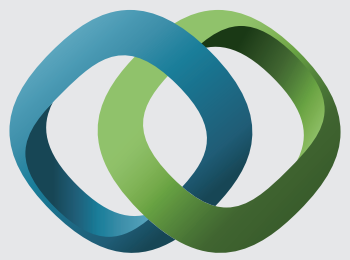

\section{Hindawi}

Submit your manuscripts at

https://www.hindawi.com
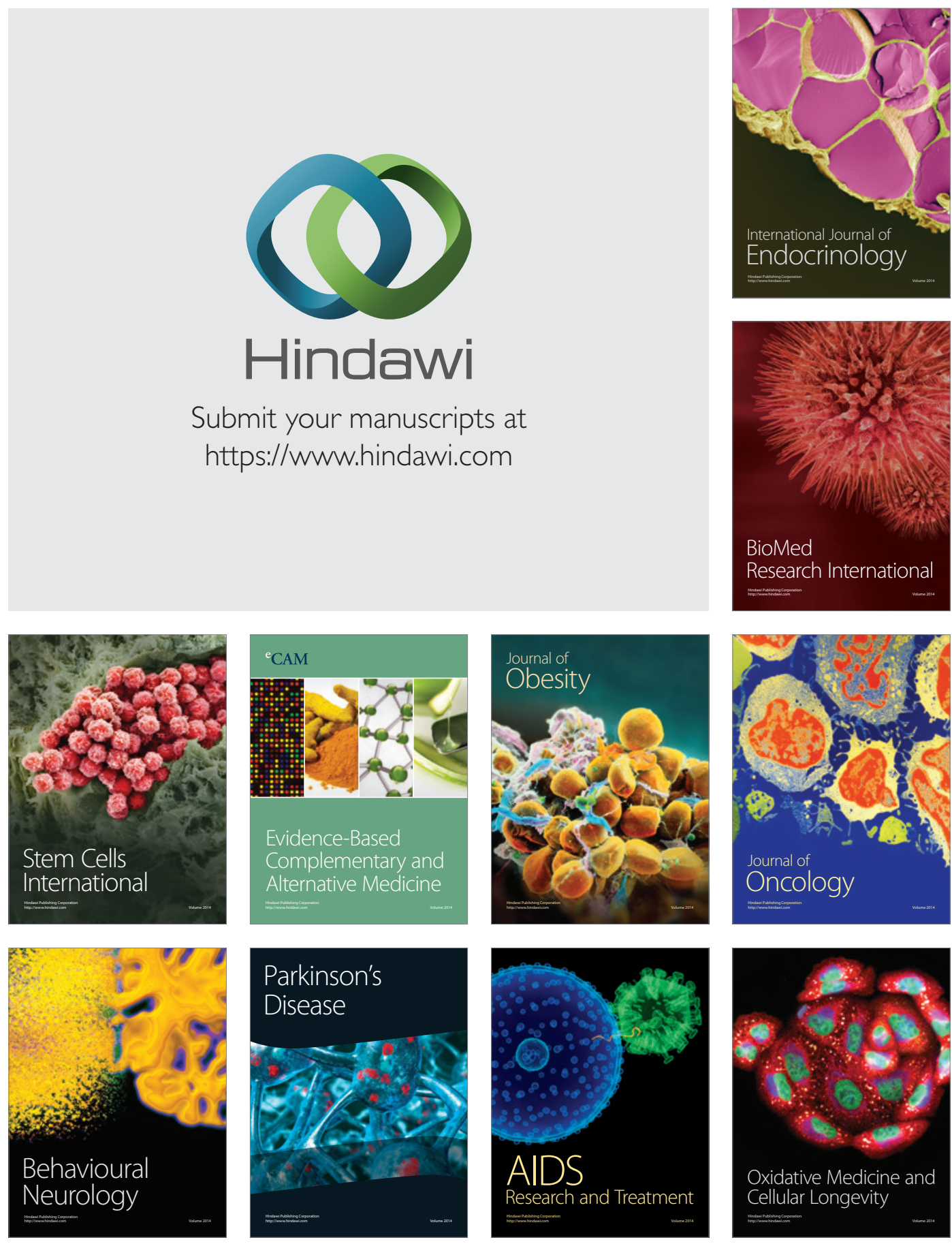